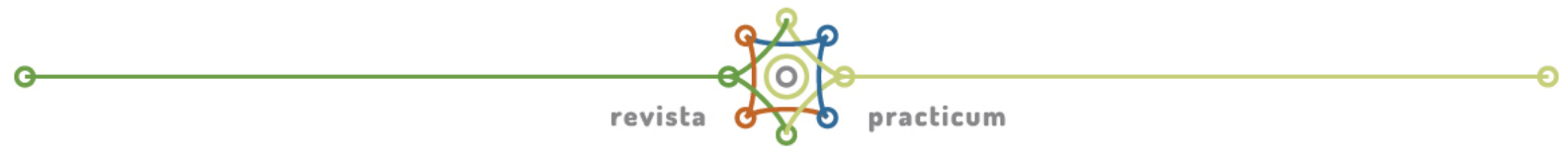

Revista Practicum, V1(1), julio-diciembre 2016

ISSN: $2530-4550$

\title{
Los modelos de formación dual como integración de lo académico y lo laboral
}

\section{Dual training models as integration of academic and labor}

\section{Dr. Sandra Lentzen}

Gerencia de formación profesional para psicoterapeutas, Wiesbadener, Akademie für Psychotherapie, Alemania. sandra.lentzen@gmx.de

Lentzen, S. (2016). Los modelos de formación dual como integración de lo académico y lo laboral. Revista Practicum, V1(1), 24-39 http://revistapracticum.com

Fecha de recepción: 07 de mayo de 2016

Fecha de revisión: 17 de mayo de 2016

Fecha de aceptación: 25 de mayo de 2016 


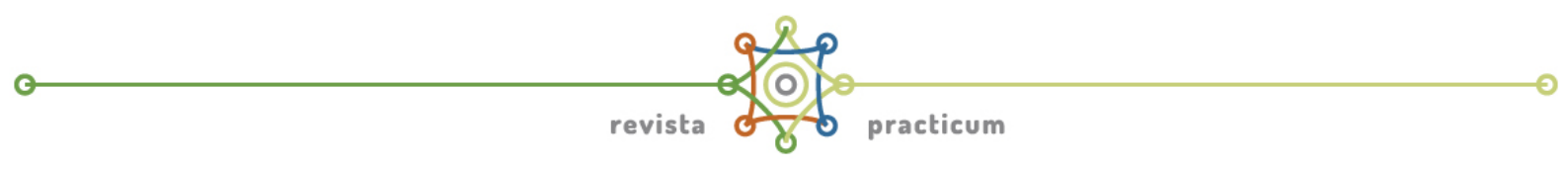

\section{Resumen}

Este artículo presenta ejemplos para la integración de la teoría y la práctica en el marco de las carreras duales en Alemania. ¿Es que la carrera dual integra satisfactoriamente la teoría con la práctica o bien la carrera dual tampoco sabe acabar con el déficit teórico-práctico del cual sufren las carreras no duales? En la carrera dual ofrecida por las universidades, escuelas superiores y academia de formación profesional (Berufsakademien) en Alemania, la demanda por una permeabilidad caracterizada por formatos y estructuras híbridas se refleja de forma especial por la distribución equilibrada entre la formación académica, la teoría y la práctica laboral. En el artículo se presenta primero la formación dual dentro del sistema académico de Alemania. A continuación, se presenta el ejemplo de una estudiante, Inés Maier, en su camino de profesionalización teóricopráctico en sus estudios duales en una Academia de formación profesional. Fue conducida una entrevista autobiográfica-narrativa, sus diarios prácticos y académicos tanto como sus casos prácticos fueron analizados. Como conclusión, la interconexión estrecha entre la teoría y la práctica acompaña, refleja y fomenta tanto la realización del conocimiento científico en la práctica (Dewe 2009) como la formación de una autoconciencia profesional.

\section{Abstract}

This article is dealing with the question, how integration and the interaction between theory and practice function in Germany within the framework of dual courses of studies. Isn't the deficit in practice, complained about when it comes to graduates of universities who did not take part in dual courses of studies, best being met my the integration of theory and practice in dual courses of studies? Dual courses of studies, in Germany offered by universities, colleges of higher education and universities of cooperative education, have a particular permeability. It is characterised by the training's format and the hybrid structures between academic education, theory and practical education. First of all, the essay illustrates the dual system within the framework of the academic systems in Germany. Afterwards, a theoretical-practical professionalisation of a real student - let's call her Inés Müller - is being traced on her path in a dual course of studies at a university of cooperative education. Methodically, this is being carried out by an autobiographical-narrative interview, by homework and term papers. The result is, that a dual course of studies represents a very good connection between theory and practice, to develop a pedagogical professionalism on a theoretical-practical basis as well as to prevent the ,praxis shock' when reality hits. 


\section{Palabras claves}

Carrera/ formación dual, relación entre teoría y práctica, autobiografía narrativa, investigación cualitativa

\section{Keywords}

Dual enrollment, theory practice relationship, personal narratives, qualitative research

\section{Cambio del panorama académico en Alemania: el desa- rrollo de la formación dual}

En la formación dual que suelen ofrecer en Alemania las universidades, las escuelas superiores y las Academias de formación profesional, que son escuelas superiores que combinan los estudios con la formación profesional, la demanda por una permeabilidad marcada por formatos y estructuras híbridas se ve reflejada especialmente por la distribución equilibrada entre la formación académica - la teoría - y la práctica laboral. La meta principal de la formación dual es llegar a una cualificación doble de las tituladas y titulados, ampliando la cualificación científica por una cualificación práctica.

Durante los últimos 40 años las carreras duales se han establecido dentro del panorama de las universidades y escuelas superiores en Alemania como el formato de formación que combina la práctica laboral con la enseñanza científica. No obstante con un $6 \%$ de todos los tipos de formación universitaria, el formato de la carrera dual actualmente ocupa un nicho dentro de la amplia oferta de formación secundaria en Alemania. Contemplando los años 2012 y 2013, el número de carreras duales de la formación vocacional primaria aumentó en un 11,4 por ciento. Las escuelas superiores públicas y privadas ofrecen un $59 \%$ de todas las carreras duales. Las academias de formación profesional ofrecen un $15 \%$ de las carreras duales (BiBB 2013, 26 y siguientes). Siguen siendo pocas las universidades que ofrecen una carrera dual, sólo un $3 \%$ en el caso de la formación vocacional primaria (véase Goeser 2013).

Al contemplar el conjunto de las 1014 carreras duales, la mayor parte, con un $43 \%$, la ofrecen los departamentos universitarios de las ciencias económicas, seguida por la oferta por parte de los departamentos de la ingeniería $(40 \%)$ y de la informática $(12,2 \%)$. No obstante se ha creado un número notable de carreras duales nuevas en los ámbitos del trabajo social, de la sanidad y la asistencia sanitaria (Goeser 2013). En el año 2013 contábamos en el ámbito del trabajo social con unas 41 carreras duales. Esto equivalía a un aumento de un $32 \%$ en comparación con el año 2012 (BiBB 2013, p. 31). 


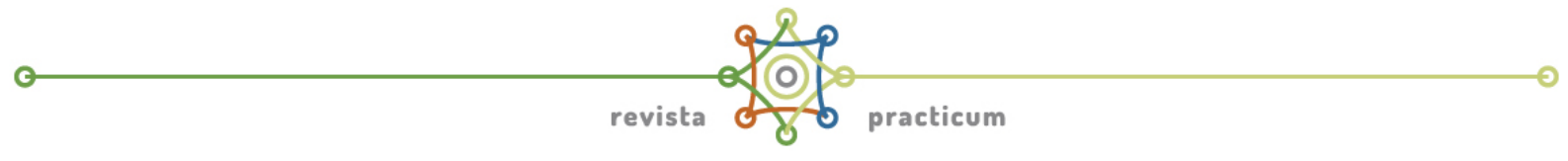

Se puede dar por hecho que dentro de la política científica la importancia de la formación dual está aumentando y aumentará en el futuro de forma especial. Esto se debe a su función de interfaz dentro del sistema educativo así como a la demanda especialmente elevada por parte de los estudiantes y de las empresas. Adicionalmente, el modelo de la carrera dual ganó de atractivo por los diversos acuerdos alcanzados por la conferencia alemana de ministros de Educación y Ciencia, entre ellos la decisión sobre la equivalencia de las Academias de formación profesional y las escuelas superiores así como la decisión acerca de la equiparación legal de las carreras que terminan con el título de grado ofrecidas por las Academias de formación profesional por un lado y por las universidades tanto como las escuelas superiores por el otro lado (KMK 2004).

\section{2. ¿Cómo podemos caracterizar la formación dual?}

El término "formación dual" o "carrera dual" describe un formato de estudios que combina (muy) (estrechamente) la práctica laboral con una formación científica. La carrera dual es fruto del desarrollo de la formación dual dentro del sistema de la formación profesional. Combina los elementos del sistema de la formación laboral y del sistema universitario mientras que liga de forma armonizada y sistemáticamente los lugares de formación. Así que la formación dual combina dos lugares de formación: por un lado la universidad, la escuela superior o la academia de formación profesional, las cuales se ocupan de proporcionar el conocimiento teórico o académico. Por otro lado el período práctico, durante el cual los estudiantes suelen aplicar sus conocimientos teóricos en la vida laboral, se realiza en una empresa, o sea en una institución o en una organización que se encarga de la formación práctica. Durante el transcurso de toda la carrera se ligan y se compaginan de forma muy estrecha los temas teóricos y prácticos a enseñar.

"En concreto significa que las universidades y las empresas han de trabajar juntos - dentro del marco establecido para elaborar los currículos, para garantizar que haya, aparte de los docentes, expertos en las empresas que se ocupen de la formación vocacional de los estudiantes, y para que las empresas cubran los gastos de la formación práctica y les paguen una remuneración a los estudiantes" (Graf et al 2014, 11).

Tras el concepto de la "formación dual" encontramos una gran variedad de carreras que se distinguen tanto con respecto a su formato como a sus estructuras (Wissenschaftsrat 2013, 7). Existen "cuatro más dos" modelos de la formación dual. 
(1) La formación dual con aprendizaje integrado combina la formación teórica con el aprendizaje en una profesión con formación vocacional autorizada. De esta forma se vinculan los períodos de estudios y de la enseñanza vocacional tanto de forma temporal así como igualmente en cuanto a los temas que se enseñan. Además, el estudiante termina esta carrera con dos títulos: el título universitario y el título profesional.

(2) La formación dual con práctica integrada combina los estudios universitarios con un período más bien largo en una empresa. Entre las clases de la universidad y la formación práctica hay una vinculación con respecto a los temas curriculares. El pre-requisito para poder inscribirse a una carrera con práctica integrada es que el estudiante haya conseguido un contrato legal con una empresa, sea este un contrato laboral, un contrato de prácticas o un contrato de voluntariado. El período práctico en la organización tiene, en comparación con las carreras tradicionales con prácticas, una importancia mucho mayor. Además, el período práctico ofrece una vinculación más estrecha con la universidad o las Academias de formación profesional. Y el lugar de la enseñanza práctica suele organizar el período práctico del estudiante de forma parecida a una formación profesional o un aprendizaje. Por esta razón la empresa es también un así llamado "lugar de enseñanza".

El primer y el segundo modelo son en general ofertas para la formación vocacional primaria. La oferta está destinada a personas con título de enseñanza media que permite acceder a la educación superior, a interesados capacitados para una carrera superior y a personas con experiencia profesional. Durante los últimos años las carreras con práctica integrada han experimentado un aumento mayor en comparación con las carreras con aprendizaje integrado (BiBB 2012). El éxito del modelo que integra la práctica se debe a que de esta forma se pueden estudiar dualmente aquellas carreras que no disponen de un aprendizaje propiamente dicho. Este es el caso en el ámbito social, la sanidad, la educación y la asistencia sanitaria. Son rubros que muestran una gran escasez de especialistas, la cual se sabe combatir con medidas provenientes del ámbito académico, entre ellas esta nueva formación teórica-práctica que incluye un título universitario altamente reconocido.

La formación dual que integra o acompaña una profesión es una formación suplementaria, adecuada para aquellas personas que quieren seguir una carrera mientras continúen trabajando en sus respectivas profesiones.

(3) La formación dual con profesión integrada combina el estudio con el ejercicio de una profesión a tiempo parcial. 


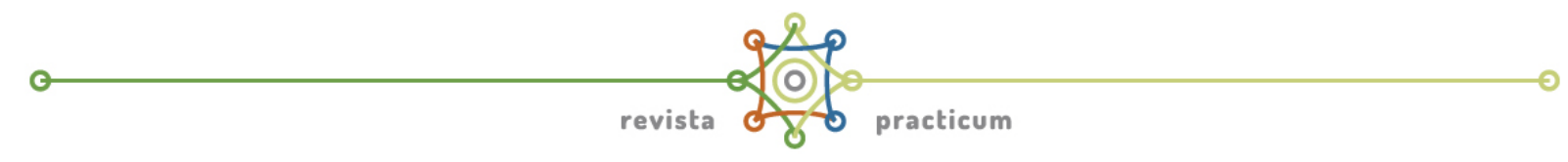

(4) La formación dual acompañante al ejercicio profesional es semejante a la carrera a distancia (Wissenschaftsrat 2013,8).

(5) En el caso de la formación acompañada por prácticas, los estudios ocupan más espacio que la práctica. El período práctico no está vinculado con la formación universitaria ni de forma institucionalestructural ni en cuanto al currículo (Wissenschaftsrat 2013, 9).

(6) En el caso de la formación con práctica integrada se vinculan las fases prácticas más sistemáticamente y de mayor impacto con la formación universitaria, en comparación con las carreras con práctica obligatoria.

De esta forma los diversos tipos de carreras duales se distinguen sobre todo en cuanto al grado de vinculación entre sus currículos y a continuación en cuanto al grado de vinculación entre los lugares de enseñanza: el lugar de la enseñanza práctica y el lugar de la enseñanza académica. La existencia de dos lugares de enseñanza es lo que tienen en común los diferentes tipos de formación dual.

La idea de la formación dual era la de atraer a aquellas personas, que ya cuentan con una formación profesional o / y cierta experiencia profesional. Eso no se ve reflejado por los datos disponibles. Según estos datos, la mayoría de los estudiantes que empiezan una carrera dual, tiene el título de enseñanza media que permite acceder a la educación superior. Como no se recogen en un lugar único las cifras de los diferentes tipos de formación dual ni el número de estudiantes, se nos presenta una situación poco satisfactoria en Alemania en cuanto a las cifras oficiales. Tampoco existe ninguna definición clara en cuanto a la formación dual. Adicionalmente a esta borrosidad de definición y la falta de datos, en Alemania hay una diversidad enorme de marcos legales y formales para la formación dual, que siempre depende de cada Estado federado (Wissenschaftsrat 2013, 16). 


\section{3. ¿Entonces, cuáles son las características de una carre- ra dual?}

A pesar de la borrosidad de definición, el Wissenschaftsrat ${ }^{1}$ ha emitido unos criterios para que en el marco de este concepto se puedan clasificar mejor la teoría y la práctica (2013, 24 siguiente).

- La relación, coordinación y vinculación de los lugares de enseñanza: La formación dual combina a partes iguales una formación académica y científica con los elementos de una formación profesional. Los lugares de enseñanza académica y de la enseñanza práctica deben ser vinculados por currículo, así como temporal e institucionalmente, por el nivel de armonización entre los temas de la enseñanza y de los módulos y por la integración del período práctico en el plan de estudios como su convalidación dentro del marco del Sistema Europeo de Transferencia y Acumulación de Créditos - ECTS.

- La exigencia científica y el grado de su realización: Los criterios de esta dimensión son el tamaño de la participación académica, los requisitos científicos de la oferta académica y la concordancia con las respectivas carreras tradicionales. El período de enseñanza académica ha de cubrir, como mínimo, un 50\% del período de la enseñanza completa. La enseñanza académica ha de otorgar unos dos tercios de los créditos ECTS al estudiante.

- La organización de la orientación práctica y de la enseñanza práctica: Dentro de esta dimensión se puede especificar cuán intensivo y cuán largo es la enseñanza práctica. De igual importancia es la pregunta, cómo acompañamos el aprender práctico. El Consejo de acreditación ha elaborado unos requisitos obligatorios para la asignación de créditos ECTS durante el período práctico: los períodos prácticos han de ser integrados en la formación y supervisados por la universidad o la Academia de formación profesional. La Academia de formación profesional organiza los períodos prácticos y define los currículos, así como en el caso necesario se entablan seminarios académicos que acompañen temáticamente a cada fase práctica.

- Las contribuciones por parte de la empresa: Esta dimensión investiga la forma y la perspectiva con la cual la empresa se ocupa de los estudiantes. Entre ello contamos por ejemplo el tipo

\footnotetext{
${ }^{1}$ El Consejo Académico de La República Federal de Alemania (Wissenschaftsrat) es el gremio alemán que aconseja al Estado federal y a los Estados federados en todas las cuestiones acerca del sistema universitario y el fomento estatal de instituciones de investigación.
} 


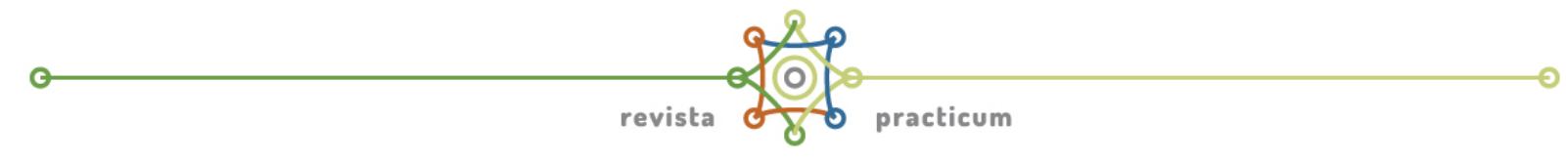

de empleo. La dualidad de la formación se basa en los acuerdos obligatorios entre las partes colaboradores. El estudiante firma un contrato con la universidad o la Academia de formación profesional y con su respectiva institución encargada de su formación práctica. Adicionalmente existe una vinculación contractual entre la universidad / la Academia de formación profesional y la institución encargada de la formación práctica.

- Las contribuciones por parte de la Academia de formación profesional: Esta dimensión describe la oferta por parte de la universidad más allá de la oferta formativa. Ésta incluye por ejemplo la oferta de asistencia social para los estudiantes o los elementos concretos de vinculación durante los módulos, como por ejemplo un seminario para reflexionar la práctica y para trabajar los informes prácticos.

- Costes y financiamiento: Los criterios son, si se paga la matrícula y hasta qué nivel las empresas participan en la financiación de la formación académica.

- ¿Cómo se realiza la dualidad en la Academia de formación profesional International (IBA) y en especial en la carrera "educación social y gestión" que está cursando la estudiante Inés Maier?

\section{La carrera "educación social y gestión "de la IBA: un ejemplo para la formación dual}

La Academia de formación profesional International (IBA) es - dentro del sector terciario de educación - una entidad de enseñanza privada y ofrece la formación de grado en el modelo de la semana partida.

Inés Maier está estudiando la carrera de educación social y gestión, una carrera dual con práctica integrada. A lo largo de la semana laboral se realizan tanto fases de teoría como de práctica. En cada semana Inés pasa 20 horas en clases de la IBA y 20 horas en una institución colaboradora donde adquiere sus conocimientos prácticos. Todo el período práctico entonces equivale a un trabajo a tiempo parcial de unas 20 horas semanales. De esta forma y a lo largo de los tres años y medio de esta carrera, los estudiantes realizan unas prácticas que equivalen a unos 21 meses de trabajo a tiempo completo. Durante toda la carrera ambos lugares de la enseñanza, tanto la empresa como la Academia de formación profesional, tienen estrechos lazos sistemáticos. La carrera "educación social y gestión" de la IBA esta acreditada hasta el año 2017.

Formalmente, la carrera tiene una duración de 7 semestres y al obtener un total de 210 créditos ECTS los estudiantes finalizan la carrera 
con el título de Grado. Los 210 créditos ECTS se dividen en 180 créditos de educación social y trabajo social y 30 créditos de gestión. De esta forma cada estudiante recibe junto a los conocimientos socioeducativos también sus primeros conocimientos en el ámbito de la gestión, fundamento necesario para estar preparado para luego asumir un puesto de trabajo en la dirección de las instituciones socioeducativas.

Si en un primer paso evaluamos esta carrera mediante las dimensiones presentadas anteriormente para caracterizar la formación dual: ¿la carrera cumplirá con los requisitos de la teoría de las dimensiones tal como la recomienda el Consejo Académico?

Hablamos primero de la dimensión "relación, coordinación y vinculación de los lugares de enseñanza". Con respecto a esta dimensión, la cual me gustaría no separarla completamente en la práctica de los demás dimensiones dejemos hablar primero a la estudiante Inés Maier mediante su entrevista autobiográfica-narrativa:

"Si, entonces yo aporto muy a menudo y con frecuencia ideas, es decir, impulsos de la teoría a la práctica. Y a veces era también desde la práctica a la teoría. Por ejemplo en el tema de las evaluaciones. Así que, en aquel entonces no tenía idea de qué es eso. Y cuando nosotros tuvimos la clase que trataba sobre las personas que fueron encuestadas durante la investigación, nosotros hemos discutido sobre el tema y luego me había quedado claro de qué se trataba. Y entonces, uno empieza a analizarlo todo con la ayuda de los métodos de evaluación, que nos habían enseñado (...) Así es que, sí traigo de forma regular a mi institución lo que he aprendido en la IBA. O viceversa, cuando yo pregunto algo en clase y por ello aprendo las bases teóricas de todo lo práctico" (L. 175-199).

Trasferir las ideas de la teoría a la práctica como llevar las preguntas de la realidad laboral a las clases de la IBA, Inés muestra unos ejemplos para este traslado teórico-práctico. Entre ellos se encuentra el siguiente: en el segundo semestre Inés asistió a un seminario que abarcaba el tema de la investigación de los encuestados, la investigación de los usuarios así como de la evaluación. Trajo a clase el cuestionario que su empresa usaba para evaluar el grado de satisfacción de sus clientes. Los resultados de las discusiones en la clase influyeron en el perfeccionamiento del cuestionario de su empresa.

Según la idea de la dualidad, esta carrera cumple con la dimensión "la exigencia científica y el grado de su realización", ya que los estudiantes pasan un $50 \%$ de su carrera en el lugar de la enseñanza académica y obtienen más de dos tercios de los créditos ECTS en este período teórico.

Por lo tanto, en lo que respecta a estas dos dimensiones, la concepción de esta carrera cumple con los requisitos establecidos por el Consejo Académico. ¿Dónde encontramos diferencias en comparación con una uni- 


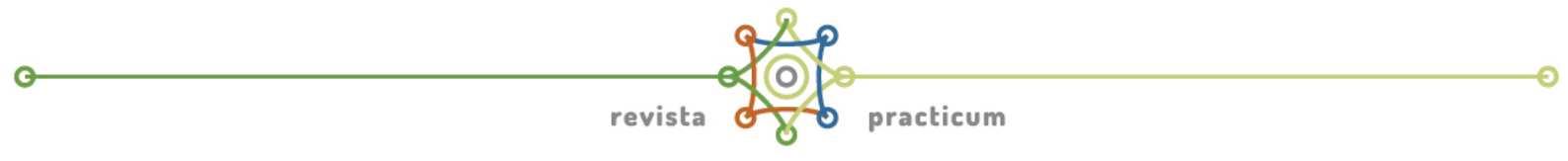

versidad? Inés es una estudiante que realizó diferentes estudios en diferentes lugares de enseñanza y por lo tanto ha ido conociendo diferentes procesos de enseñanza y de educación. Ella inició sus estudios de Derecho en el semestre de invierno del año 2010 en una universidad estatal. Inés comentó estos estudios: "y al fin y al cabo nunca estaba nadie allí en las clases. Así que yo siempre buscaba todas las cosas para toda la gente. Y cuando había llegado el momento en el cual yo necesitaba algo, claro está, entonces tuve que entender que regía una situación competitiva entre nosotros." (L. 68-70). Después de un año académico, Inés cambió de universidad: "allí seguía de forma igual. Y, bueno, entonces decidí acabar con estos estudios a mediados del semestre" (L. 72/73). Inés interrumpió sus estudios en mayo 2012 y de ahí empezó a trabajar en una institución social. "Pegaba desde el primer día" (L. 84). La institución social en la cual trabajaba en un principio de ayudante temporal, se ha convertido en la institución que se encarga de su formación práctica durante su carrera dual. Este nuevo comienzo, Inés lo comenta con las siguientes palabras: "entonces sale un poco el sol, mejor dicho, desde que empecé a trabajar en mi organización"(L. 388/389).

"Cuando yo he iniciado mis estudios en la IBA, es claro que tenía un poco el miedo de fracasar (...). Y yo he pensado que igual esto me podría pasar nuevamente. Entonces te vuelves un poco histérica... Y yo misma me he presionado bastante (...) Quiero hacerlo perfecto porque me gusta y me divierte. $Y$ creo que es bueno que yo pueda allí preguntar, que sencillamente tenga contacto con los profesores, porque sé cómo es la universidad normalmente, allí eres un número de matrícula cualquiera y ninguna persona se interesa por ti. (...) Y me parece súper bien que los profesores te animen en este camino. Sí." (L. 101-114)

Como el período práctico es parte integral de esta carrera y recibe asistencia por parte de la IBA, se ven cumplidos los requisitos con respecto a la orientación práctica en el sentido descrito en la dimensión de "la organización de la orientación práctica y de la enseñanza práctica" y junto a ello también los de la dimensión sobre "las contribuciones por parte de la universidad". Las instituciones responsables exigen que - para ser reconocidas como lugar de enseñanza práctica por parte de la IBA - la institución práctica colaboradora ofrezca las actividades suficientes en el área del trabajo social. Una vez por semestre se realiza, por ejemplo, un encuentro con los representantes de las instituciones que sirve para tratar las preguntas básicas en cuanto a la colaboración entre la IBA y las empresas. Durante el estudio los estudiantes han de elaborar unos informes prácticos. Cada informe práctico vincula un seminario teórico con el trabajo práctico. 
Las contribuciones por parte de la empresa, costes y financiamiento: La empresa o institución colaboradora paga al estudiante su matrícula más una mesada para los gastos personales. Adicionalmente las empresas pagan la asistencia social y otras prestaciones.

\section{5. ¿Por qué nos inspira la idea de la carrera dual "educa- ción social y gestión"?}

La carrera dual fortalece adquirir la competencia de acción profesional apoyándola en los resultados científicos, la trasmisión del conocimiento científico (Harmsen 2014, 93) así como en los métodos de actuación específicos de la profesión. Según Schweppe y Thole (2005), la transferencia teoría-práctica en combinación con un gran escepticismo entre los representantes de la vida laboral y los estudiantes en cuanto a la teoría, es uno de los grandes desafíos para la enseñanza. A menudo, la impresión es que el comportamiento de los investigadores y el comportamiento de los que trabajan en el ámbito del trabajo social, son poco compatibles. Da la impresión que vincular la teoría con la práctica es imposible (von Spiegel 2013). El encanto de la formación dual con práctica integrada en el ámbito social es la posibilidad de poder ofrecer alternativas para reventar estas estructuras. Al asistir estrechamente a los estudiantes desde el principio de su carrera luchamos juntos contra el "shock de la realidad" (Spatschek 2009, 212) y contra la "insuficiencia práctica de los titulados de grado" (Schröder 2010, 138). De esta forma nos enfrentamos a la así llamada distancia práctica de los titulados universitarios que tantas veces se reclama.

\section{La relación entre la teoría y la práctica en la carrera "educación social y gestión": profundicemos el ejemplo de Inés Maier}

Inés Maier trabaja los tres días prácticos de la semana en una institución de la asistencia temprana. La asistencia temprana es un sistema de asistencia regional y local en Alemania. Desde el punto teórico profesional es el mandato doble de ayuda y control que domina esta área de asistencia educativa dentro del sistema de la asistencia social a la infancia y a menores en Alemania. Dentro del dilema de la prevención, la idea universal de fomentar, ayudar y proteger a la gente lucha muchas veces contra una realidad reactiva que se basa en la intervención. La oferta de ayuda para padres y menores es un elemento central del trabajo socioeducativo y empieza con el embarazo y continúa durante los tres primeros años de la vida. Las posibilidades del desarrollo de niños y padres deben centrarse en, por ejemplo, la ayuda en asuntos prácticos de la vida cotidiana y en el fomento de las competencias relacionales y educativas. Cuando los padres perciben y evalúan de forma errónea el comportamiento de sus bebés, esto puede conducir a comportamientos muy peculiares y a situaciones de sobre-exigencia que pueden derivar fácilmente en conflictos violentos (Kinderschutz-Zentren 2016). El trabajo preventivo también significa que, mediante el reconocimiento de hitos relevantes para el peligro del interés 


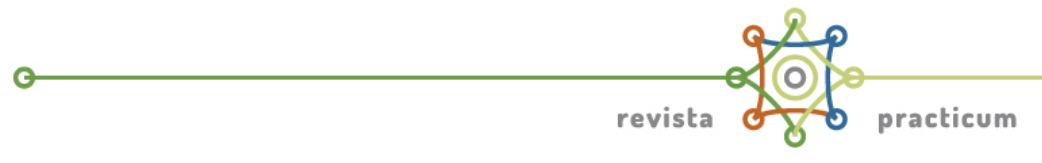

superior de los menores (§8a SGB VIII), los educadores sociales tienen el deber legal de actuar, antes de que los menores entren en una situación de riesgo. Con frecuencia estas señales son imprecisas y pueden tal vez manifestarse en la sobrecarga de los padres.

Inés ha asistido durante el tercer semestre al seminario "profesionalización pedagógica". En este seminario se lleva a cabo la provisión de conocimientos teórico-profesionales, sin que se intenta en un primer lugar, trasmitir un conocimiento. Ella relata el siguiente caso práctico:

\section{Caso: Doreen Schmidt ${ }^{2}$}

Doreen Schmidt tiene 28 años y comparte domicilio con su ex marido, Jeremy-Pascal, de 35 años. Tienen un hijo de un año y medio que se llama Justin. Se conoce poco al padre, se deja ver de vez en cuando en las fiestas del jardín de infantes. Doreen explicó en una entrevista de orientación con su educadora que sufre bulimia y que Justin come todo lo que quiere. Ella dice: «Vivo en un miedo permanente de que Justin pase hambre». Justin come excesivamente para su edad. Las educadoras deben «frenarle» de vez en cuando. Ellas dicen: «Se atiborra de comer todo lo que tiene en el plato. Justin come más que una persona adulta». En la reunión en que se trabaja este caso como caso clínico de forma anónima (con la psicóloga, la doctora y la trabajadora social), la educadora busca consejo para manejar mejor el problema. La trabajadora social es también responsable de la ayuda familiar y durante la discusión advierte en seguida que se trata de Doreen y sabiendo eso transfiere estas informaciones nuevas a la Oficina de Juventud. Unos días más tarde, Doreen recibe una carta de la Oficina de Juventud por peligro para el interés superior del menor. Se queda completamente desconcertada. Cuando la Sra. Wiegand efectúa la visita semanal a su familia, la Sra. Schmidt le habla de esta carta. La Sra. Wiegand se la queda mirando y no le puede ofrecer respuesta alguna (Maier 2014a, 10 y ss.).

Este caso práctico le preocupa mucho a Inés, ya que tiene la sensación de que la trabajadora social no ha guardado su distancia profesional

${ }^{2}$ La Literatura Maier (2014a, 2014b, 2014c) y la entrevista autobiografica pueden ser solicidadas a la autora. 
sino que se extralimitó. En su informe práctico que ha de elaborar para este seminario, Inés vincula la experiencia práctica con su conocimiento teórico sobre la profesionalidad pedagógica:

Inés empieza con las antinomias de la acción socioeducativa: La primera antinomia es la antinomia entre la organización y la interacción. En el caso de la asistencia temprana, esta contradicción de la acción pedagógica está muy presente. Por un lado hay que convencer a las familias de aceptar la oferta de ayuda mientras que siempre interfiere suavemente el cargo estatal de proteger al niño. En el sentido de una ponderación errónea del conocimiento de conducta, una "acción descalificada puede, en un caso de protección del riesgo temprano, dañar el acceso a la familia y malograr la posibilidad de una ayuda apropiada" (Maier 2014b, 4). La acción profesional para Inés incluye, apoyado por el "conocimiento científico" disponer "de conocimientos especializados para saber distinguir las diversas formas de riesgo infantil, para saber evaluar el riesgo y para conocer la efectividad de la ayuda" (Maier 2014b, 4). En la interpretación del caso distingue entre su propia comprensión profesional y la postura de la trabajadora social, que parece que no tenía una competencia reflexiva de acción ni un entendimiento más detenida del caso. La Sra. Wiegand como la trabajadora social, ya que opta por dirigirse exclusivamente a la organización de la Oficina de Juventud, no debe embarcarse en una interacción con la clienta. El proceso interactivo concreto que incluye procesos de negociación comunicativa y una estructura de acción pedagógica no apta para organizarla de forma tecnológica se ve reemplazado por estructuras organizativas rutinarias: en última instancia mediante la carta de la Oficina de Juventud sin que se haya efectuado ninguna interacción previa. Otra antinomia de este caso es la de la organización e interacción. El centro de padres y menores en el que trabajan la estudiante y la trabajadora social, no tiene, distinto a la Oficina de Juventud, función explícita de control. El margen legal del centro aparenta que, ante la sospecha del riesgo para el interés superior del menor, la acción profesional puede y debe pedir a los padres una exención de su discreción profesional. Es algo que la educadora social no pidió. La "sesión de discutir un caso anónimo" no ha cumplido con el anonimato. La trabajadora social actúa en su función de responsable para la ayuda familiar: transmite la información que ha recibido, como trabajadora social durante una reunión anónima a la persona responsable de la familia Schmidt en la Oficina de Juventud. También se da la antinomia de la proximidad y la distancia en este caso de Doreen. Inés indica que el resultado de una conducta pedagógica en el sentido de una proximidad positiva, tendría que ser la confianza (Maier 2014b, 8). La Sra. Wiegand parece haber ocupado una "distancia igualadora y conformadora del rol" (Helsper 2014, 26), negativa para Doreen. Esta conducta decepciona a la clienta en y con respecto a la interacción educativa: Doreen está "totalmente desconcertada" por la carta, así como por las medidas adoptadas por la trabajadora social.

En tanto a realidad y deseo para una conducta profesional en el ámbito de la ayuda temprana, Inés pone en su caso ejemplar otro acento 


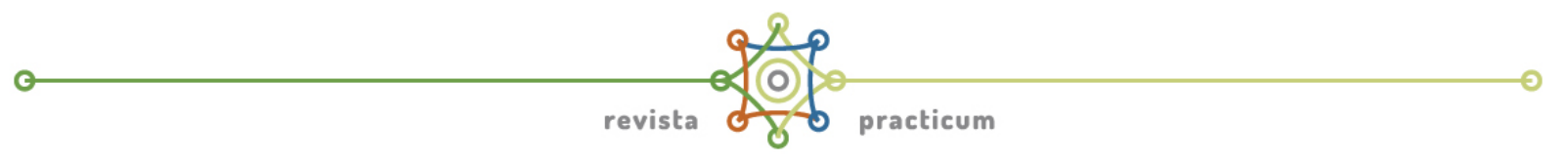

que la educadora social: Para avanzar en la protección de los niños en el sistema de la ayuda infantil y juvenil se debería acompañar y fomentar a los padres, menores y jóvenes en el desarrollo de su capacidad de autocontrol, en vez de implementar formas de control ajeno observadoras y estigmatizadoras (Maier 2014a, 13).

Poco después, Inés se ocupa nuevamente del caso (Maier 2014c), esta vez en el marco del seminario "análisis de las situaciones de acción". Este seminario pone el acento en la contemplación reflexiva de lo que ya ha pasado y de lo que está pasando actualmente. Basado en los tres niveles "poder, saber y comportamiento" de von Spiegel (2013), se analizan situaciones de acción tanto a nivel del caso particular como a nivel de un tratamiento generalizado.

Inés Maier relata que ahora, debido a la denuncia por delito contra el bienestar del niño, el tribunal había sancionado a la madre a inscribirse a una terapia contra el trastorno alimenticio de niños en forma de un curso a distancia y en línea. Era fundamental para esta sanción, que la madre había sufrido de bulimia. Se anotaba todo lo que Justin solía comer o beber. Antes de que la situación "se podía agravar" (Maier 2014c) la madre había ido en un último intento a un experto para enfermedades intestinales de niños. Este realizó una colonoscopía con la diagnosis "distrofia". Eso significaba que a Justin le gustaba comer sin que sintiera una sensación de saturación alguna. Adicionalmente no absorbía la cantidad suficiente de sustancias nutritivas por lo que él siempre sufría hambre. Las preguntas a las que se enfrentaron los estudiantes en este caso práctico y que intentaron responder a nivel teórico, eran preguntas de tal índole "¿cómo actuar de forma adecuada por parte de la pedagoga socioeducativa y del trabajo social?" Además se discutía si no se había fijado demasiado en la enfermedad de la madre al buscar una diagnosis para el hijo y si no es por eso que los profesionales habían perdido de vista otros posibles pasos de acción.

\section{Resumen: la relación entre la teoría y la práctica}

Los diferentes ejemplos que trajeron los estudiantes en los seminarios concerniendo sus vidas laborales les demuestran, que con cada caso se repite la "constelación de crisis" de la diferencia entre teoría y práctica y que cada vez han de trabajar nuevamente con el potencial de errores. Las paradojas de acción y las antinomias fundamentales han de conocerse y trabajarse reflexivamente. Sólo de este modo puede alcanzarse la competencia de acción profesional y sólo de esta manera, como también lo experimentó Inés por sus casos prácticos, la educación social se vuelve práctica y en el caso concreto cumple con su función de ayuda. La complejidad y ambivalencia de la práctica puede resultar irritante, también en el contexto de la formación de una competencia de 
acción profesional. Hay que permitirse el trabajo de reflexión para reflexionar y acumularlo desde el punto de vista científico. Crear una competencia de acción profesional solo es posible si se relacionan los fundamentos teóricos científicos con la apropiación continua y reflexiva de los conocimientos necesarios (Schütze 1993).

Los estudiantes adquieren la competencia socioeducativa de acción profesional no solo a base de conocimientos científicos o de conocimientos teóricos de la realidad del caso. El trabajador social como actor profesional formado científicamente se distingue por sus conocimientos adicionales de reflexión de los trabajadores sociales que solo erráticamente tratan con los procesos sociales. Adicionalmente, los seminarios durante los cuales se instruye y practica la capacidad de tratar de forma reflexiva los conocimientos científicos y las perspectivas desconocidas, así como aquellos que proporcionan opciones de explicación y evaluación fundamentadas científicamente, son de alta relevancia ya que ofrecen herramientas para una competencia de acción profesional. Por consiguiente, la orientación a la práctica ya durante los estudios de este modo puede fundamentar un vínculo más estrecho entre la teoría y la práctica y facilitar posibilidades específicas para la formación de una competencia de acción profesional.

\section{Referencias bibliográficas}

BIBB (Bundesinstitut Für Berufsbildung) (2012). Datenreport 2012. https://goo.gl/FS65NQ [Recuperado 15 Mayo 2016].

BiBB (Bundesinstitut für Berufsbildung) (2013). Datenreport 2013. https://goo.gl/hYcyA4 [Recuperado 15 Mayo 2016].

Dewe, B. (2009). Reflexive Professionalität: Maßgabe für Wissenstransfer und Theorie-Praxis-Relationierung im Studium der Sozialarbeit. In A. Riegler, S. Hojnik \& K. Posde, K. (Eds.), Soziale Arbeit zwischen Profession und Wissenschaft, pp. 41-63. Wiesbaden: VS Verlag.

Goeser, J. et al (2013). AusbildungPlus in Zahlen. Trends und Analysen. Bonn. https://goo.gl/JqVjpH [Recuperado 15 Mayo 2016].

Graf, L. et al (2014). Duale Studiengänge im globalen Kontext: Internationalisierung in Deutschland und Transfer nasch Brasilien, Frankreich, Katar, Mexiko und in die USA. Köln: DAAD.

Harmsen, T. (2014). Professionelle Identität im Bachelorstudium Soziale Arbeit. Wiesbaden: Springer.

Helsper, W. (1996). Antinomien des Lehrerhandelns in modernisierten pädagogischen Kulturen. Paradoxe Verwendungsweisen von Autonomie und Selbstverantwortlichkeit. In A. Combe, W. Helsper (Eds.), Pädagogische Professionalität. pp. 521-5509. Frabkfurt: Suhrkamp. 


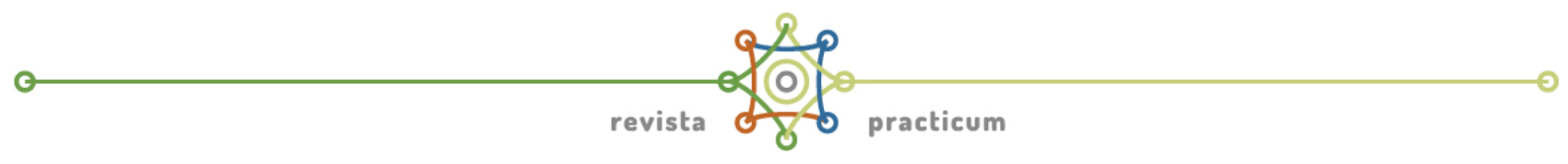

Kinderschutz-Zentren

(2016).

Kinderschutz.

Bundesarbeitsgemeinschaft der Kinderschutz-Zentren [e.v]. [Recuperado 15 Mayo 2016].

KMK (Kultusministerkonferenz) (2004). Einordnung der Bachelorausbildungsgänge an Berufsakademien in die konsekutive Studienstruktur (Beschluss der Kultusministerkonferenz vom 15.10.2004). Bonn. http://goo.gl/bzqOQp [Recuperado 15 Mayo 2016].

Schröder, H. (2010). Was sollen sie können? - Die aktuelle Fachdebatte über Kompetenzen von Fachkräften mit Bachelor- und Masterabschlüssen in der Kinder und Jugendhilfe. Soziale Passagen, 2, 135-142.

Schütze, F. (1993). Die Fallanalyse: zur wissenschaftlichen Fundierung einer klassischen Methode der Sozialen Arbeit. In T. Rauschenbach, F. Ortmann, M.-E. Karsten (Eds.), Der sozialpädagogische Blick: lebensweltorientierte Methoden in der Sozialen Arbeit, pp. 191-221. Weinheim: Juventa Verl.

Schweppe, C., Thole, W. (eds). (2005). Sozialpädagogik als forschende Disziplin. Weinheim \& München: Juventa Verlag.

Spatschek, C. (2009). Use After Reading. Einschätzungen zum Stand der Theorieentwicklung in der Sozialen Arbeit, zu ihren aktuellen Perspektiven sowie den daraus entstehenden Herausforderungen für die Lehre. In B. Birgmeier, E. Mührel, E. (Eds), Die Sozialarbeitswissenschaft und ihre Theorie(n). Positionen, Kontroversen, Perspektiven, pp. 209-217. Wiesbaden: VS Verlag.

Von Spiegel, H. (2013). Methodisches Handeln in der Sozialen Arbeit. Stuttgart: UTB Verlag.

Wissenschaftsrat (2013). Empfehlungen zur Entwicklung des dualen Studiums. http://goo.gl/pGfzOU [Recuperado 15 Mayo 2016]. 